\title{
Impact of COVID-19 pandemic and lockdown on emergency room access in Northern and Central Italy
}

\author{
Fabrizio Giostra, ${ }^{1}$ Maria Grazia Mirarchi, ${ }^{1}$ Gabriele Farina, ${ }^{1}$ Ciro Paolillo, ${ }^{2}$ Catello Sepe, \\ Francesca Benedusi, ${ }^{2}$ Andrea Bellone, ${ }^{3}$ Lorenzo Ghiadoni, ${ }^{4}$ Massimo Santini, ${ }^{4}$ Greta Barbieri, ${ }^{4}$ \\ Grazia Guiddo, ${ }^{5}$ Alessandro Riccardi, ${ }^{5}$ Roberto Lerza, ${ }^{5}$ Daniele Coen ${ }^{6}$ \\ ${ }^{1}$ Emergency Room and Emergency Medicine, Ospedale Sant'Orsola, Bologna; ${ }^{2}$ Emergency Room and \\ Emergency Medicine, Ospedali Civili, Brescia; ${ }^{3}$ Emergency Room and Emergency Medicine, Ospedale \\ Niguarda, Milan; ${ }^{4}$ Emergency Room and Emergency Medicine, Azienda Ospedaliera Universitaria di Pisa; \\ ${ }^{5}$ Emergency Room and Emergency Medicine, Ospedale San Paolo, Savona; ${ }^{6}$ Academy of Emergency \\ Medicine and Care (AcEMC), Milano, Italy
}

\begin{abstract}
In Northern Italy the coronavirus infection has spread since February 2020: the increase in admissions of COVID-19 patients corresponded to a drastic decrease in admissions of regular patients to the Emergency Room (ER). This retrospective study
\end{abstract}

\author{
Correspondence: Alessandro Riccardi, Emergency Room and \\ Emergency Medicine, Ospedale San Paolo, Via Genova 70, 17100 \\ Savona, Italy. \\ Tel.: +39.0198.404963 \\ E-mail: dottriccardi@gmail.com
}

Key words: Overcrowding; COVID-19; triage; emergency room; Italian epidemic.

Contributions: MGM and GF wrote the Materials and Methods section; $\mathrm{CP}, \mathrm{CS}$, and FB co-wrote the Discussion section; MS, and GB co-wrote the Introduction section; GG and AR wrote the Results section and the References section; FG, RL, DC, AB and LG reviewed the paper and co-wrote all sections. All authors endorse this paper and contribute to his realization

Conflict of interest: None. This work was not supported by any grant. FG, CP, AB, LG, GB, and DC are members of the editorial board of Emergency Care Journal.

Availability of data and materials: All data generated or analyzed during this study are included in this published article.

Ethics approval and consent to participate: This retrospective paper was authorized by our medical directions, and does not involve patients

Consent for publication: Non applicable.

Received for publication: 25 February 2021.

Revision received: 27 March 2021

Accepted for publication: 6 April 2021.

This work is licensed under a Creative Commons Attribution 4.0 License (by-nc 4.0).

(C) Copyright: the Author(s), 2021

Licensee PAGEPress, Italy

Emergency Care Journal 2021; 17:9705

doi:10.4081/ecj.2021.9705 was conducted by Academy of Emergency Medicine and Care (AcEMC). During the lockdown period the accesses were reduced by more than $50 \%$, and in the following months of May and June 2020 , there was a recovery clearly below $(70 \%)$ previous year's numbers. We have observed a drastic reduction in white and green codes, a fair reduction in yellow codes, while red codes remained stable. The decrease in access to the ER mainly concerned patients with low priority color codes, but also the reduction in the number of accesses of yellow and red codes, insignificant at a superficial glance, is notable. If we consider that yellow and red codes during the months of the lockdown included many patients with COVIDrelated respiratory insufficiency, it is evident that there was a clear reduction in the number of serious illnesses not COVID-related. This is certainly another serious consequence of the COVID-19 pandemic.

\section{Introduction}

The coronavirus pandemic has deeply changed people's ways of life. These changes also significantly affected the use of health services, especially by non-infected individuals. ${ }^{1,2}$ In Northern Italy the infection has spread rapidly since the end of February 2020, and especially in the months of March and April, hospitals had to admit many patients affected by COVID-19. The large increase in admissions of COVID-19 patients corresponded, at the same time, to a drastic decrease in admissions of regular patients to the emergency room. ${ }^{3-6}$ This was certainly the result of instructions from health authorities not to approach the emergency room except for extreme necessities. Legal provisions ${ }^{7}$ (lockdown, quarantine, social distancing, domestic isolation) and the fear of being infected also played an important role in this phenomenon. This study was conducted by the Academy of Emergency Medicine and Care (AcEMC) with the aim of analysing this phenomenon and measuring quantitatively and qualitatively which type of patients avoided going to the emergency room during this time period.

\section{Materials and Methods}

This retrospective study was conducted by collecting emergency department accesses for March and April 2020 (corresponding to the lockdown in Italy) ${ }^{7}$ and the following two months. All data collected were compared with the same periods in 2019. Emergency department accesses from five hospitals in Northern 
and Central Italy were analyzed using computerized systems. These are five city hospitals (Bologna, Brescia, Milan, Pisa, Savona) with emergency departments as reported in the authors' affiliation that together receive about 35,000 patients each month, corresponding to the heterogeneous geographical spread of the COVID-19 epidemic. ${ }^{8}$ The parameters measured were i) the total number of accesses and their subdivision by triage priority code, ii) the total number of hospitalizations from the emergency department and the admissions to the cardiology department, iii) the number of triage accesses by general macro-diagnosis such as trauma and burns, chest pain and abdominal pain.

\section{Results}

The approximately 35,000 emergency room visits per month that we analyzed with reference to 2019 were distributed in percentage terms for $28 \%$ to Sant'Orsola hospital in Bologna, $23 \%$ to Niguarda hospital in Milan, 19\% to Brescia hospital, 19\% in Pisa and $11 \%$ in Savona. Figure 1 shows that during the lockdown period those accesses were reduced by more than $50 \%$, and that in the following months of May and June 2020, there was a recovery clearly below (70\%) previous year's numbers. In addition, we can see a drastic reduction in white and green codes, a fair reduction in yellow codes, while the number of red codes remained stable. Figure 2 shows how total admissions from the emergency depart-

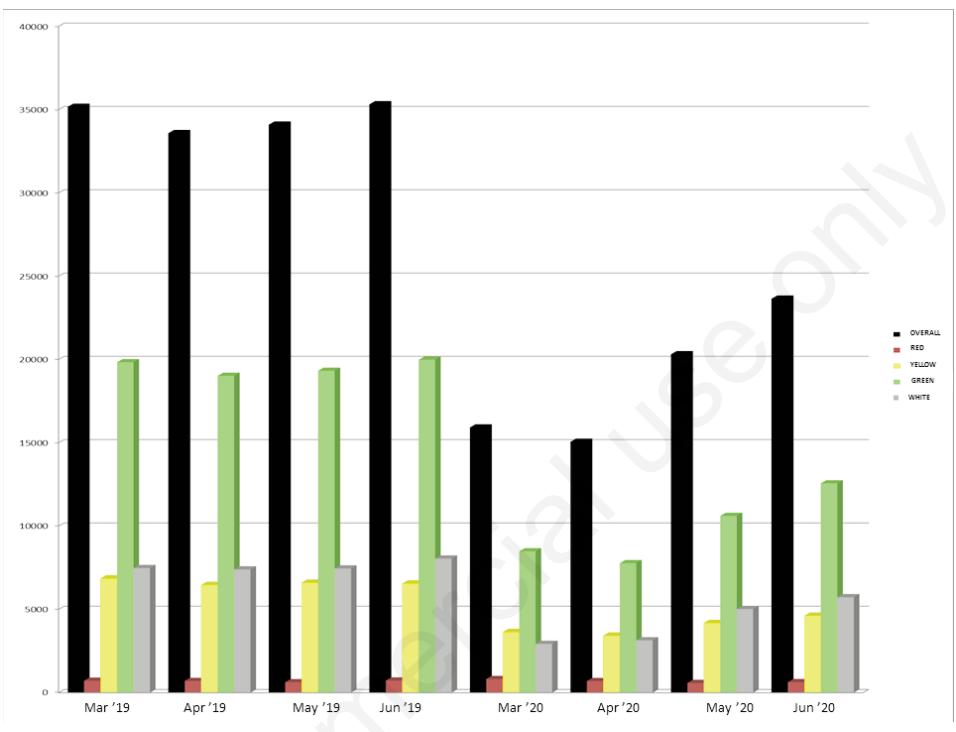

Figure 1. Emergency room visits and triage code.
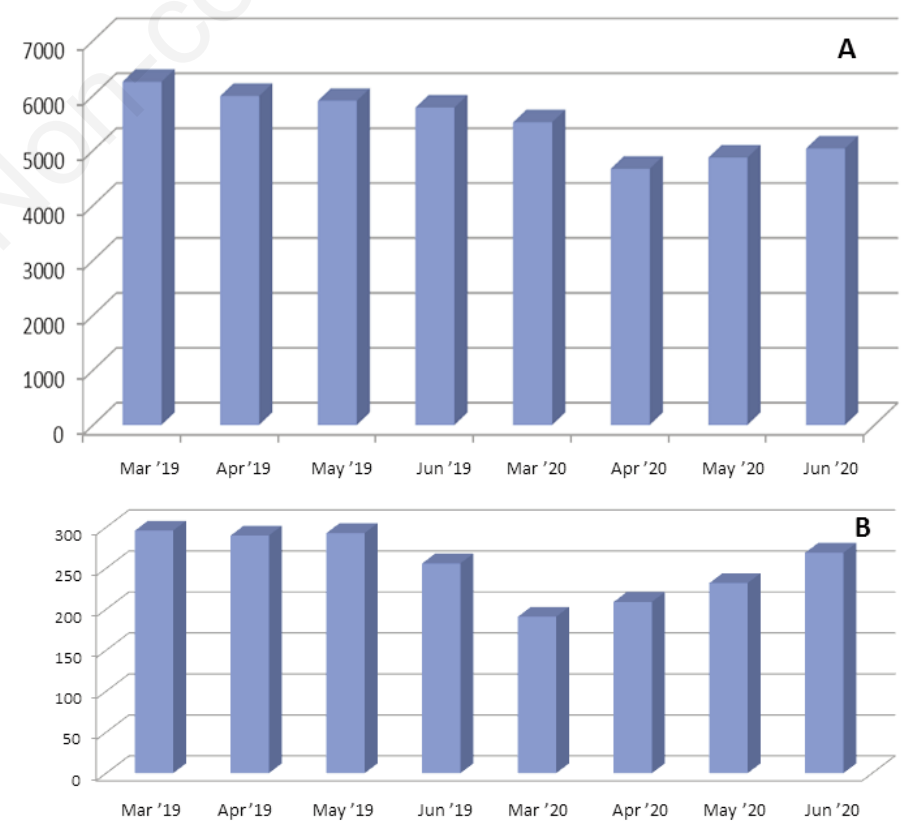

Figure 2. A) Total hospitalizations from the emergency room; B) Hospitalizations in cardiology. 
ment in the two months of the lockdown were reduced by approximately 20\% compared to the same months in 2019 (Figure 2A) and even more marked (30\%) is the percentage reduction in admissions to cardiology (Figure 2B). Certainly, the most important data are evident in Figures 3 and 4. The reduction in accesses due to trauma during the lockdown period is the most marked (about $80 \%$, Figures 3 ) but the reduction in patients with abdominal pain (Figures $4 \mathrm{a}$ ) and chest pain (Figures $4 \mathrm{~b}$ ) was also very important (about 70 and $60 \%$ respectively). All the measured parameters (Figures 1 to Figures 4) showed a progressive increase in the two months following the lockdown, however without reaching the values of the same months in 2019.

\section{Discussion}

The data submitted unequivocally demonstrate that the COVID-19 pandemic caused a sharp decline in access to the emer- gency room, especially during the lockdown period (Figure1). ${ }^{1,2}$ This reduction was similar in all 5 structures analyzed. Their geographical distribution suggests that the phenomenon was probably the same throughout the country. This is also to be expected because the reasons were similar in every ED of different regions, despite heterogeneous geographical spread of the COVID-19 epidemic in Italy. ${ }^{8}$ First of all, the lockdown and its consequences, but also the fear of getting infected inside hospitals and the recommendation not to crowd hospital services. The decrease in access to the emergency room mainly concerned patients with low priority color codes, i.e., white and green codes. It is obvious that the reduction in the number of inappropriate and non-serious cases is the main reason for the increase in the percentage of hospitalizations from emergency rooms. The absolute number of hospitalizations decreased during the pandemic periods, but their percentage went from $15-20 \%$ to around $30 \%$ of accesses to the emergency room. However, this study highlights other important changes in the use of emergency services. The most significant reduction, but also the

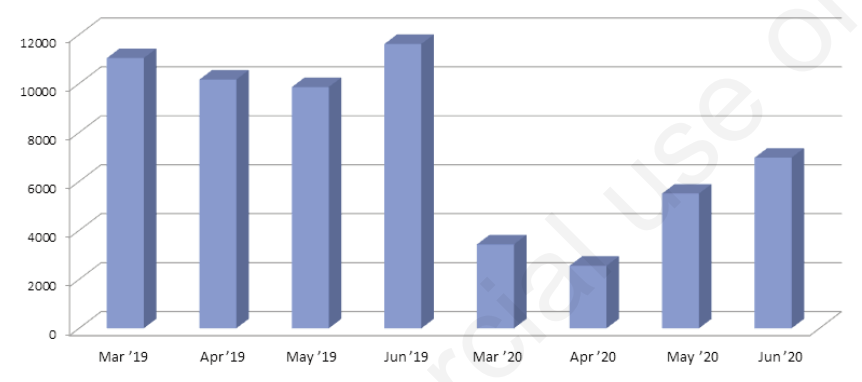

Figure 3. Visits for trauma or burns.

Figure 4. A) Visits for abdominal pain; B) Visits for chest pain. 
most obvious, was seen in the counts of trauma as triage macrodiagnosis (Figure 3). This observation can be easily explained, because the lockdown actually determined an absolute reduction in the number of traumatic events. Traffic bans, severe restrictions on leaving home, and the cancellation of sports events and many work activities have had a significant impact on the number of road, sports and work-related accidents. Less marked than for trauma, but still very significant, were decreases in accesses for chest pain (Figure 4B) and abdominal pain (Figure 4A) in the months of lockdown (Figure 4A-B). This deserves some consideration because within triage macro-diagnoses, such as chest pain and abdominal pain, potentially dangerous clinical situations are hidden with considerable frequency. The lockdown may not have reduced the absolute number of these diseases. Some patients with minor illnesses might certainly have decided not to go to the emergency room for fear of being infected. However, unfortunately we believe that some serious clinical situations also behaved in the same way. This can be seen from the reduction in the absolute number of admissions (Figure 2A) and particularly in cardiology (Figure 2B), but also from the reduction in the number of accesses of yellow and red codes, which may appear insignificant at a superficial glance (Figure 1). In fact, if we consider that yellow and red codes during the months of the lockdown included many patients with COVID-related respiratory insufficiency, it is evident that there was a clear reduction in the number of serious illnesses not COVID-related. This is certainly another serious consequence of the COVID -19 pandemic and a cause for concern, because it means that during these critical months many patients suffering from acute and severe illnesses opted not to turn to the emergency system, with the potential consequences of delays in diagnosis and treatment. This is consistent with the observation of De Filippo et $a l .{ }^{9}$ regarding the reduction in hospital admissions for acute coronary syndrome during the COVID-19 epidemic in Northern Italy. Our observation shows how the pandemic found our health service unprepared. It is unthinkable and unacceptable that a citizen should choose not to go to the emergency service for fear of worsening his or her health by contracting a disease. This must be the prerequisite for major but necessary changes in our organisation, ${ }^{10-14}$ especially in terms of training health workers and their numbers, which must be more substantial, the separation of pathways for infected and non-infected patients within the emergency department, and therefore the architecture of hospitals and emergency facilities, which must be designed with patient and worker safety in mind. In parallel telemedicine should be to be implemented to ascertain adequate follow-up to chronic cardiovascular disease at risk of (re-) hospitalization for acute complications. On the other hand, these data show a substantial change in the use of ED by population, clearly reducing their presence in the Hospital, raising the issue of a large number improper use of the ED for diseases that are not really urgent.

\section{References}

1. Jeffrey MM, D’Onofrio G, Paek H, et al. Trends in emergency department visits and hospital admissions in health care systems in 5 states in the first months of the COVID-19 pandemic in the US. JAMA Int Med 2020;180:1328-33.

2. Rosenbaum L. The untold toll: the pandemic's effects on patients without COVID-19. N Eng J Med 2020;382:2368-71.

3. Barbieri G, Spinelli S, Filippi M, et al. COVID-19 pandemic management at the Emergency Department: the changing scenario at the University Hospital of Pisa. Emerg Care Journal 2020;16:9146.

4. Comelli I, Scioscioli F, Cervellin G. Impact of the COVID-19 epidemic on census, organization and activity of a large urban emergency department. Acta Biomed 2020;91:45-9.

5. Poggiali E, Vercelli A, Cillis MG, et al. Triage decision-making at the time of COVID-19 infection: the Piacenza strategy. Intern Emerg Med 2020;15:879-82.

6. Turcato G, Zaboli A, Pfeifer N. The COVID-19 epidemic and reorganisation of triage, an observational study. Intern Emerg Med 2020;15:1517-1524.

7. Decree of the President of the Council of Ministers of the Italian Government on March 9, 2020. Available from: https://www.gazzettaufficiale.it/eli/id/2020/03/09/20A01558/s $\mathrm{g}$

8. Istituto Superiore di Sanità. Sorveglianza integrata COVID-19 in Italia. 2020. www.iss.it/documents/20126/0/Infografica 09marzo.pdf/1f62ad0a-e156-cf27-309d-26adcb1b52b4?t=1 583782049035

9. De Filippo O, D’Ascienzo F, Angelini F. et al, Reduced rate of hospital admissions for ACS during COVID-19 outbreak in Northern Italy. N Eng J Med 2020;383:88-9.

10. Coen D, Paolillo C, Cavazza M, et al. Changing Emergency Department and hospital organization in response to a changing epidemic. Emerg Care J 2020;16:8969.

11. Poggiali E, Vercelli A, Mazzoni S, et al. COVID-19 pandemic, piacenza calling. The survival strategy of an Italian emergency department. Acta Biomed 2020;91:1-3.

12. Maniscalco P, Poggiali E, Quattrini F, et al. The deep impact of novel COVID-19 infection in an Orthopedics and Traumatology Department: the experience of the Piacenza Hospital. Acta Biomed 2020;91:97-105.

13. Carenzo L, Costantini E, Greco M, et al. Hospital surge capacity in a tertiary emergency referral centre during the COVID19 outbreak in Italy. Anaesthesia 2020;75:928-34.

14. Turcato G, Zaboli A, Pfeifer N. The COVID-19 epidemic and reorganisation of triage, an observational study. Intern Emerg Med 2020;15:1517-24. 